\title{
Hunting the vector hybrid
}

\author{
A. Donnachie* \\ TH Division, CERN, 1211 Geneva 23, Switzerland
}

Yu. S. Kalashnikova

ITEP, 117259 Moscow, Russia

(Received 2 February 1999; published 3 November 1999)

\begin{abstract}
The current state of analysis of $e^{+} e^{-}$annihilation below $2.0 \mathrm{GeV}$ and of the vector component of $\tau$ decay is reviewed. The evidence for and against the presence of hybrid vectors is discussed. It is concluded that the data strongly favor their inclusion, and the consequences of this are outlined. [S0556-2821(99)02021-4]

PACS number(s): 12.39.Mk, 12.39.Jh, 13.25.Jx
\end{abstract}

\section{INTRODUCTION}

It has been recognized for some time that the data on vector meson decays appear to be in conflict with the predictions of the ${ }^{3} P_{0}$ model which has become the standard for calculating meson decays. One solution has been to suggest that the physical light vectors are mixed $q \bar{q}$ and hybrid vectors, as the latter have appropriate decay characteristics. However this has never been quantified, nor have alternative non-hybrid explanations been actively sought. Here we explore the limits of the ${ }^{3} P_{0}$ model and apply a specific non${ }^{3} P_{0}$ model to the vector decays in an attempt to avoid the introduction of hybrids. Constraints placed on these models by other decays, in particular those of the pseudoscalars $\eta(1295)$ and $\pi(1300)$, are sufficient to prevent them from providing a solution to the vector decay problem. Given that the inclusion of hybrids is unavoidable, we consider the advantages and disadvantages of the flux tube model and the constituent gluon model of hybrids in the context of the vector decays. The data prefer the constituent gluon model, and we outline briefly the consequences elsewhere of this choice.

The current information on light-quark vectors from $e^{+} e^{-}$annihilation and $\tau$ decay is discussed in Sec. II, and the decay problems identified. Present understanding of gluonic excitations in general and of hybrid models in particular is summarized in Sec. III. In addition to the standard ${ }^{3} P_{0}$ approach, which models the string breaking, we suggest a specific hadronic ansatz for relevant light quarkonia decays in analogy to the decays of heavy quarkonia. These two approaches are evaluated in the context of pseudoscalar decays, specifically $\eta(1295)$ and $\pi(1300)$, in Sec. IV and limits put on the corresponding vector decays. These latter results are confronted with the data in Sec. V, where it is shown that the limits are too restrictive to resolve the problems identified in Sec. II. This leads naturally to a detailed consideration of the two available models for hybrids, and the data appear to favor the constituent gluon model over the flux tube model. Our conclusions and their consequences are summarized in Sec. VI.

\footnotetext{
*Permanent address: Department of Physics and Astronomy, University of Manchester, Manchester M13 9PL, England.
}

\section{II. $e^{+} e^{-}$ANNIHILATION AND $\tau$ DECAY}

The existence of the isovector $\rho(1450)$ and $\rho(1700)$, and their isoscalar counterparts $\omega(1420)$ and $\omega(1600)$ is now well established [1]. The key experimental results in determining the existence of the two isovector states were $e^{+} e^{-}$ $\rightarrow \pi^{+} \pi^{-}[2]$ and $e^{+} e^{-} \rightarrow \omega \pi[3]$. These original data sets have subsequently been augmented by data on the corresponding charged channels in $\tau$ decay [4,5], to which they are related by conservation of vector current (CVC). These new data confirm the earlier conclusions. The data on $e^{+} e^{-} \rightarrow \pi^{+} \pi^{-} \pi^{+} \pi^{-}$[6] and $e^{+} e^{-} \rightarrow \pi^{+} \pi^{-} \pi^{0} \pi^{0} \quad[6,7]$ (excluding $\omega \pi$ ) and the corresponding charged channels in $\tau$ decay [5] are compatible with the two-resonance interpretation $[8,9]$. However the $4 \pi$ data alone do not provide such good discrimination despite $4 \pi$ being the major decay channel. The reason for this is straightforward. In $\omega \pi$ and $\pi \pi$ there is strong interference with the tail of the $\rho$, which is absent in the case of $4 \pi$. It was also found that the $e^{+} e^{-}$ $\rightarrow \eta \pi^{+} \pi^{-}$cross section is better fitted with two interfering resonances than with a single state [10], with parameters in fair agreement with those found in the analysis of other channels. Independent evidence for two $J^{P}=1^{-}$states was provided in a high statistics study of the $\eta \pi \pi$ system in $\pi^{-} p$ charge exchange [11]. Decisive evidence for both the $\rho(1450)$ and $\rho(1700)$ in their $2 \pi$ and $4 \pi$ decays has come from the study of $\bar{p} p$ and $\bar{p} n$ annihilation [12]. The masses and widths obtained from the analysis of the $2 \pi$ channel are in reasonably good accord with those found from $e^{+} e^{-}$annihilation and $\tau$ decay. The significance of these results for the observation the vector mesons via their $4 \pi$ decay is that they represent a combined analysis of four high-statistics channels: $\bar{p} p \rightarrow 5 \pi^{0}, \bar{p} d \rightarrow \pi^{-} 4 \pi^{0}(p), \bar{p} p \rightarrow \pi^{+} \pi^{-} 3 \pi^{0}$ and $\bar{p} d \rightarrow \pi^{+} 2 \pi^{-} 2 \pi^{0}(p)$. The inclusion of both the $\rho(1450)$ and the $\rho(1700)$, with masses and widths fixed at the values obtained in the $2 \pi$ analysis, leads to a significant improvement in $\log (L)$.

The data initially available for the study of the corresponding isoscalar states $\omega(1420)$ and $\omega(1600)$ were $e^{+} e^{-}$ $\rightarrow \pi^{+} \pi^{-} \pi^{0}$ (which is dominated by $\rho \pi$ ) and $e^{+} e^{-}$ $\rightarrow \omega \pi^{+} \pi^{-}$[13]. The latter cross section shows a clear peak which is apparently dominated by the $\omega(1600)$. The former cross section shows little structure, but is appreciably larger than that calculated from the tails of the $\omega$ and $\phi$. This im- 
TABLE I. Experimental masses and widths of the higher vector mesons.

\begin{tabular}{rrrrr}
\hline \hline Resonance & $\rho(1450)$ & $\rho(1700)$ & $\omega(1420)$ & $\omega(1600)$ \\
\hline Mass $(\mathrm{MeV})$ & $1465 \pm 25$ & $1700 \pm 20$ & $1419 \pm 31$ & $1649 \pm 24$ \\
Width $(\mathrm{MeV})$ & $310 \pm 60$ & $240 \pm 60$ & $174 \pm 59$ & $220 \pm 35$ \\
\hline \hline
\end{tabular}

plies an additional contribution and a best fit is obtained with two states [8], although a fit with only the $\omega(1600)$ cannot be excluded completely. Data on $\bar{p} N$ annihilation may help to clarify the situation, but analysis is still at a preliminary stage [14].

Although there is general consensus on the existence of the $\rho(1450), \rho(1700), \omega(1420)$ and $\omega(1600)$ there is considerable disparity on the parameters of these resonances. These show variation from one reaction to another and, even within one particular process, are dependent on the analysis techniques employed. Results from channels for which there is strong interference with the tail of the $\rho$ or of the $\omega$ and $\phi$ are sensitive to the choice of model used to estimate this contribution. For the $\rho(1450)$ the most extreme low mass comes from an analysis of the $\pi^{+} \pi^{-}$spectrum in the reaction $K^{-} p \rightarrow \pi^{+} \pi^{-} \Lambda$ [15], which gives $1266 \pm 14 \mathrm{MeV}$. However such a low mass is not supported by any other analysis and does require confirmation. Most of the results of the analyses of $e^{+} e^{-}$annihilation, $\tau$ decay and $\bar{p} N$ annihilation are clustered round the preferred Particle Data Group (PDG) values [1], which are the ones we use here. These are given in Table I.

A natural explanation of these states is that they are the first radial, $2{ }^{3} S_{1}$, and first orbital, $1{ }^{3} D_{1}$, excitations of the $\rho$ and $\omega$ as the masses are close to those predicted by the quark model [16]. This interpretation is given further credence by the observation of $\phi(1680)$ which has the appropriate mass to be a candidate for the first radial excitation of the $\phi$.

Despite the reasonable agreement of the observed masses with the quark model predictions, the ratio of the $e^{+} e^{-}$ width of the $\rho(1700)$ to that of the $\rho(1450)$ is surprisingly large. In the non-relativistic limit the $e^{+} e^{-}$width of the $1^{3} D_{1}$ state vanishes, and although some non-zero width will be created by relativistic corrections this is expected to be small. Additionally the data on the $4 \pi$ channels in $e^{+} e^{-}$ annihilation and in $\tau$ decay do not appear at first sight to be compatible with those expected for the vector radial and orbital excitations of the $q \bar{q}$ system. This statement is of course model dependent as it assumes that we can predict the hadronic decays of the vector $q \bar{q}$ excitations. The ${ }^{3} P_{0}$ model [17-21] does appear to allow this with some accuracy. A systematic study of known light $q \bar{q}$ decays shows that a ${ }^{3} P_{0}$-type amplitude dominates, and widths which are predicted to be large or small are found respectively to be so. More quantitatively, calculated widths agree with data to within $25-40 \%$. Of course the accuracy of the ${ }^{3} P_{0}$ model has only been tested on specific well-known decays and it may be that its accuracy elsewhere is less certain. However there is no hard evidence to support this latter conjecture,
TABLE II. The ${ }^{3} P_{0}$ partial widths for $\rho_{2 S}$ and $\rho_{1 D}$.

\begin{tabular}{lrrrrrrrrr}
\hline \hline Channel & $\pi \pi$ & $\pi \omega$ & $\rho \eta$ & $\pi h_{1}$ & $\pi a_{1}$ & $\rho \rho$ & $\rho \sigma$ & Other & Total \\
\hline$\rho_{2 S}$ & 68 & 115 & 18 & 1 & 3 & 10 & 1 & 80 & 295 \\
$\rho_{1 D}$ & 27 & 23 & 13 & 104 & 105 & 6 & 0 & 137 & 415 \\
\hline \hline
\end{tabular}

and as far as one can ascertain the ${ }^{3} P_{0}$ model is reliable.

Thus the success of the ${ }^{3} P_{0}$ model for well-known decays can be used to justify its application to predicting other decays, and in particular those of the radial and orbital excitations of the $\rho$ and $\omega$. In its simplest form the ${ }^{3} P_{0}$ model contains only two parameters: an inverse length scale $\beta$ which controls the meson form factors, and the pair creation strength $\gamma$. These are not known precisely, but are reasonably well constrained with $\beta \sim 0.4 \mathrm{GeV}, \gamma=0.39$. Assuming that their masses are respectively 1.45 and $1.70 \mathrm{GeV}$, the ${ }^{3} P_{0}$ partial widths for $\rho_{2 S}$ and $\rho_{1 D}$ are given in Table II.

In Table II "other', includes $K \bar{K}, K^{*} \bar{K}+$ c.c. and $6 \pi$ channels, and the $\sigma$ is the broad $S$-wave $\pi \pi$ enhancement, considered as the $1{ }^{3} P_{0} q \bar{q}$ state. Altogether 16 channels have been incorporated in the calculation in which we have not used the narrow-width approximation for final meson states, so the results differ slightly but not significantly from those that do [21].

It is not necessary to go through a detailed analysis to show that these ${ }^{3} P_{0}$ model results exclude interpreting the $e^{+} e^{-}$and $\tau$ decay data in terms of the $\rho_{2 S}$ and $\rho_{1 D}$ if the model is strictly applied. As already implied, the key is in the $4 \pi$ decays. From Table II one can see that the $4 \pi$ decays of the $\rho_{2 S}$ are negligible, and so the $\rho_{2 S}$ effectively makes no contribution to the $4 \pi$ channel. In contrast the $4 \pi$ decays of the $\rho_{1 D}$ are large, and the two dominant ones, $h_{1} \pi$ and $a_{1} \pi$, are comparable. Now $h_{1} \pi$ contributes only to the $\pi^{+} \pi^{-} \pi^{0} \pi^{0}$ channel in $e^{+} e^{-}$annihilation, but $a_{1} \pi$ contributes to both this and to $\pi^{+} \pi^{-} \pi^{+} \pi^{-}$. An immediate consequence is that we would expect $\sigma\left(e^{+} e^{-} \rightarrow \pi^{+} \pi^{-} \pi^{0} \pi^{0}\right)$ $>\sigma\left(e^{+} e^{-} \rightarrow \pi^{+} \pi^{-} \pi^{+} \pi^{-}\right)$, after subtraction of the $\omega \pi$ cross section from the total $\pi^{+} \pi^{-} \pi^{0} \pi^{0}$. This contradicts observation. Despite considerable uncertainty in the $\pi^{+} \pi^{-} \pi^{0} \pi^{0}$ cross section, enhanced by the need to subtract the $\omega \pi$ cross section, it is undeniably appreciably smaller than the $\pi^{+} \pi^{-} \pi^{+} \pi^{-}$cross section over most of the relevant energy range.

One explanation of this has been to suggest that the $q \bar{q}$ vector states are mixed with a hybrid vector $[22,23]$ as this decays predominantly to $a_{1} \pi$ in flux tube models [23], and to $a_{1} \pi$ and $\rho(\pi \pi)_{S}$ in constituent gluon models [24]. Both the $\pi^{+} \pi^{-} \pi^{+} \pi^{-}$and the $\pi^{+} \pi^{-} \pi^{0} \pi^{0}$ channels are accessed by the $a_{1} \pi$ and $\rho(\pi \pi)_{S}$ decays so, in either case, $e^{+} e^{-}$ annihilation and the corresponding $\tau$ decays should in principle be explicable in terms of some suitable combination of $\rho, \rho_{2 S}, \rho_{1 D}$ and hybrid $\rho_{H}$, and with the implication that there must be very little $\rho_{1 D}$ to ensure the dominance of $\pi^{+} \pi^{-} \pi^{+} \pi^{-}$over $\pi^{+} \pi^{-} \pi^{0} \pi^{0}$. The surprisingly large ratio of the $e^{+} e^{-}$widths is also a good indicator of mixing.

However such evidence as we have from the isoscalar states indicates that the picture might not be quite as simple 
TABLE III. The ${ }^{3} P_{0}$ widths for the $\omega_{2 S}$ and $\omega_{1 D}$.

\begin{tabular}{lcrrrrr}
\hline \hline Channel & $\rho \pi$ & $\omega \eta$ & $b_{1} \pi$ & $\omega \sigma$ & Other & Total \\
\hline$\omega_{2 S}$ & 328 & 12 & 1 & 8 & 36 & 385 \\
$\omega_{1 D}$ & 101 & 13 & 371 & 0 & 53 & 561 \\
\hline \hline
\end{tabular}

as this. The ${ }^{3} P_{0}$ widths for the $\omega_{2 S}$ and $\omega_{1 D}$ are given in Table III [21] assuming that their masses are respectively 1420 and $1650 \mathrm{MeV}$.

The large widths of the bare states predicted by the ${ }^{3} P_{0}$ model are well in excess of the quoted experimental total widths [1]. There must be strong mixing in the isoscalar channel as the $e^{+} e^{-}$widths of the $\omega_{1}^{\prime}$ and $\omega_{2}^{\prime}$ are almost the same, and one would not expect either the $\omega_{1 D}$ or the $\omega_{H}$ to have an electromagnetic coupling comparable to that of the $\omega_{2 S}$. In the flux tube model the width of the $\omega_{H}$ is predicted to be small, $\sim 20 \mathrm{MeV}$ [23], and is essentially all to $\rho \pi$. The $\omega_{H}$ width can be appreciably larger in constituent gluon models [24] but again the $\rho \pi$ decay dominates although some $\omega(\pi \pi)_{S}$ decay is allowed [24]. Thus omitting the $\omega_{1 D}$, in analogy with the isovector case, would seem difficult to reconcile with the integrated cross section for $e^{+} e^{-}$ $\rightarrow \omega \pi \pi$ which, up to $1.8 \mathrm{GeV}$, is about $60 \%$ of the integrated $e^{+} e^{-} \rightarrow \rho \pi$ cross section and could be taken to imply some significant $\omega_{1 D}$ component.

The arguments relating to the $\rho(\pi \pi)_{S}$ and $\omega(\pi \pi)_{S}$ decays of $\rho_{2 S}$ and $\omega_{2 S}$ presuppose that there is no mechanism which can generate these in any significant way. A possible approach is to invoke an inherent uncertainty in the ${ }^{3} P_{0}$ model when applied to the decays of radial excitations to the ground state plus an $S$-wave $\pi \pi$ pair. In the ${ }^{3} P_{0}$ model the decays of the $\rho_{2 S}$ to $\rho(\pi \pi)_{S}$ and of the $\omega_{2 S}$ to $\omega(\pi \pi)_{S}$ are strongly suppressed by a cancellation between two terms, one of which is strongly dependent on the model parameters. If these decays could be sufficiently enhanced within the structure of the model then the $4 \pi$ problem in the isovector sector and the comparatively large $\omega \pi \pi$ in the isoscalar sector could possibly be resolved. Note that the $\rho(\pi \pi)_{S}$ and $\omega(\pi \pi)_{S}$ decays of the $\rho_{1 D}$ and $\omega_{1 D}$ respectively are strictly forbidden in the ${ }^{3} P_{0}$ model.

Further, many radial excitations are known to decay preferentially to the ground state, or a lower radial excitation, plus $(\pi \pi)_{S}$. The most obvious ones occur in higher quarkonia. The branching fractions of these decays are $\psi(2 S)$ $\rightarrow \psi(1 S), \quad 50.8 \pm 3.7 \% ; \quad Y(2 S) \rightarrow Y(1 S), \quad 27.3 \pm 1.4 \%$; $\mathrm{Y}(3 S) \rightarrow \mathrm{Y}(1 S), 6.5 \pm 0.4 \%, \mathrm{Y}(3 S) \rightarrow \mathrm{Y}(2 S), 4.8 \pm 0.7 \%$. These decays cannot proceed via string breaking and are all specifically non- ${ }^{3} P_{0}$ decays. The relevance of this is the implication that the $(\pi \pi)_{S}$ state couples rather strongly to gluons. It has been suggested [25] on the basis of QCD sum rules that the $\sigma$-meson is a low-lying scalar glueball and an estimate [26] of its coupling to light quarks indicates that it is strong. Thus it is not surprising that a similar phenomenon is seen in light quarkonia: for example $\eta^{\prime}(1295) \rightarrow \eta(\pi \pi)_{S}$ and $\pi(1300) \rightarrow \pi(\pi \pi)_{S}$, assuming for the moment that both $\eta^{\prime}(1295)$ and $\pi(1300)$ are radial excitations. Both of these latter decays are essentially zero in the ${ }^{3} P_{0}$ model with stan- dard parameters. Whether a similar mechanism is operating here as for heavy quarkonia, or whether these decays arise from the sensitivity of the ${ }^{3} P_{0}$ model for radial decays involving $(\pi \pi)_{S}$, is undetermined.

Before applying these various ideas to the vector meson decays we consider the current status of hybrid mesons and of radial decays to the corresponding ground state plus $(\pi \pi)_{S}$ in light quarkonia.

\section{HYBRID MESONS}

Evidence for the excitation of gluonic degrees of freedom has emerged in several processes. There are two independent indications of an isovector $J^{P C}=1^{-+}$exotic resonance $\hat{\rho}(1600)$ in $\pi^{-} N \rightarrow \pi^{+} \pi^{-} \pi^{-} N$, specifically in the $\rho^{0} \pi^{-}$ channel. The E852 Collaboration [27] quote a mass of $1593 \pm 8 \mathrm{MeV}$ and width of $168 \pm 20 \mathrm{MeV}$, which are consistent with the preliminary claim of the VES Collaboration [28] of a resonance at $1620 \pm 20 \mathrm{MeV}$ with a width of 240 $\pm 50 \mathrm{MeV}$. There is also evidence for this state in the $\eta^{\prime} \pi$ channel $[28,29]$. It has been argued that the $\rho \pi, \eta^{\prime} \pi$ and $\eta \pi$ couplings of this state support the hypothesis that it is indeed a hybrid meson, although other interpretations cannot be eliminated entirely [30]. A peak in the $\eta \pi$ mass spectrum at $1.4 \mathrm{GeV}$ with $J^{P C}=1^{-+}$, in the reaction $\pi^{-} N \rightarrow \eta \pi^{-} N$, has also been interpreted as a resonance [31]. Additional evidence for the same state in the same mode is provided by the Crystal Barrel Collaboration [32], in an analysis of $p \bar{p}$ $\rightarrow \eta \pi^{+} \pi^{-}$. In this case the signal is deduced from a phase variation in the $J^{P C}=1^{-+}$amplitude seen as interference in the Dalitz plot. There is evidence from the VES Collaboration [33] for two isovector $0^{-+}$states in the mass region 1.4 to $1.9 \mathrm{GeV}$. One is the well-established $\pi(1800)$ [34] with a mass of $1790 \pm 6 \pm 12 \mathrm{MeV}$ and width of $225 \pm 9 \pm 15 \mathrm{MeV}$, and one a new state, the $\pi(1600)$, with a mass of $1580 \pm 43$ $\pm 75 \mathrm{MeV}$ and width of $450 \pm 60 \pm 100 \mathrm{MeV}$ [33]. The quark model predicts only one state in this mass region. Thus there is evidence for degrees of freedom beyond $q \bar{q}$, and the unusual decay pattern of the $\pi(1800)$ encourages the belief that it has a strong hybrid component $[21,23]$.

The interpretation of the peak in the $\eta \pi$ mass spectrum at $1.4 \mathrm{GeV}$ as a resonance [31] has been challenged [35]. It was shown that the E852 $\eta \pi$ peak and phase can be obtained without the need to invoke the presence of an exotic resonance. The two key ingredients are the presence of a strongly coupled threshold in this mass region (taken to be $b_{1} \pi$ ) with rescattering to produce the $\eta \pi$ signal. A Deck-type background interfering with a hybrid resonance of higher mass, for which the $\hat{\rho}$ at $1.6 \mathrm{GeV}$ is an obvious candidate, was considered as the production mechanism. The Deck mechanism also provides the predominant natural parity exchange for the $1.4 \mathrm{GeV}$ peak which is observed experimentally, in contrast to the $1.6 \mathrm{GeV}$ state which has a significant contribution from unnatural parity exchange. Of course the Deck mechanism is not applicable to the $p \bar{p}$ annihilation experiment [32], but the strongly-coupled threshold with rescattering can generate sufficient phase variation without requiring a resonance at $1.4 \mathrm{GeV}$ [36].

If the above is the correct interpretation of the $\eta \pi$ data 
then the $\hat{\rho}(1600)$ is the lowest mass exotic hybrid. An alternative viewpoint is to accept that the $1.4 \mathrm{GeV} \eta \pi$ signal really is an exotic resonance [24] and to explore the consequences. One of these, which will be relevant for our subsequent discussion, is that the $J^{P C}=0^{-+} \pi(1300)$ is predominantly a hybrid meson, although there may be some admixture of the $q \bar{q} 2{ }^{1} S_{0}$ state which has the same quantum numbers. One argument usually given in favor of the hybrid interpretation is a large $\pi(\pi \pi)_{S}$ branching fraction, much larger than $\pi \rho$, although as we shall see in Sec. IV B there is considerable experimental disagreement on this point. A large $\pi(\pi \pi)_{S}$ width would be in complete disagreement with the predictions for the $q \bar{q} 2 S$ level in the ${ }^{3} P_{0}$ model for which the $\pi(\pi \pi)_{S}$ mode is strongly suppressed. However if the $\pi(1300)$ is a $q \bar{q}$ radial excitation then, as noted in Sec. II, the decay is very sensitive to the ${ }^{3} P_{0}$ parameters. The decay may also be generated by a non- ${ }^{3} P_{0}$ mechanism. Thus the $\pi(\pi \pi)_{S}$ decay may be possible without invoking a hybrid. This possibility will be explored fully in Sec. IV.

Ideally we would have mass predictions for hybrids comparable to those for the $q \bar{q}$ states. Unfortunately the absolute mass scale for light-quark hybrids is not precisely determined, with predictions for the lightest hybrids lying between 1.3 and $1.9 \mathrm{GeV}$. Bag models [37,38] tend towards the lower end of this range, but it is not clear just how reliable their results are. Parameters are tuned to fit the $q \bar{q}$ spectrum and it is questionable whether the same parameters should be used for the $q \bar{q} g$ states. Attempts to accommodate this lead to considerable variation in the predictions, giving a mass value for the lightest hybrid in the range 1.4 to $1.7 \mathrm{GeV}$. However it is perfectly possible to accommodate an $0^{-+}$at $\sim 1.3 \mathrm{GeV}$ and an $1^{-+}$at $\sim 1.4 \mathrm{GeV}$ with the lightest $1^{--}$ hybrid at $\sim 1.65 \mathrm{GeV}$. Flux tube models [39] predict hybrid masses to be considerably higher than these, at about 1.9 $\mathrm{GeV}$. The constituent gluon model [40] gives the light-quark hybrid mass at 1.7-1.8 GeV. In principle QCD sum rules could resolve the issue of mass scale as they are a powerful tool for the understanding of hadron properties in terms of the vacuum condensates of QCD. However even here there is a major divergence of view, either giving an upper limit of $1.5 \mathrm{GeV}$ on the mass of the $1^{-+}$hybrid [41], with a preference for a somewhat lower value, or putting it somewhere in the range 1.6 to $2.1 \mathrm{GeV}$ [42], with a preference for the upper end. The principal difference between the two calculations is the application of a low-energy theorem in [41] which in turn gives an important role to the $g^{3}\left\langle G^{3}\right\rangle$ term with the effect of lowering the mass. This is acknowledged in [42]. Applications of lattice gauge theories to the lightquark hybrid sector [43-46] give essentially consistent results for the mass of the $1^{-+}$hybrid, predicting it to be around $2 \mathrm{GeV}$, although admittedly with quite large errors. Indeed they are still sufficiently large that this mass is considered not to be inconsistent with the hybrid interpretation of the $\hat{\rho}(1600)$ [44].

However it does seem to be generally agreed that the mass ordering is $0^{-+}<1^{-+}<1^{--}<2^{-+}$. This is certainly the case for bag models [37,38], and also appears to hold in the heavy-quark sector [47]. The same mass ordering emerges if one assumes that the splittings are due to the spin-spin contact interaction [24]. The heavy-quark expansion of QCD in Coulomb gauge [48] demonstrates that spinorbit splitting of low-lying hybrids with $J^{P C}=J^{-+}$and $J^{+-}$ is such that $J=1$ lies between $J=0$ and $J=2$, and the ordering is the same for both sets. However either of $J=0$ or $J$ $=2$ can be the lowest-lying. In lattice QCD calculations of heavy-quark hybrid states it is found that $0^{+-}<2^{+-}[49]$ so that combining the two results gives $0^{-+}<1^{-+}<2^{-+}$(and $0^{+-}<1^{+-}<2^{+-}$). Whether it makes sense to extrapolate these heavy-quark results to the light quark sector is debatable, but nonetheless the qualitative agreement with the bag and constituent model results is encouraging. It has to be noted that in the light-quark sector it is found that nominally $2^{+-}<0^{+-}$[44]. However the errors on the masses, which are much greater relative to the central values than in the heavy-quark sector, are still sufficiently large for this result not to be considered definitive.

The lack of precision in mass estimates is matched by uncertainty on decay modes. Again the two standard approaches are the constituent gluon model $[24,50]$ and the flux tube model $[23,39]$. In the former the hybrids are considered specifically as having three components: quark, antiquark and gluon. Decays proceed by dissociation of the constituent gluon [50]. In the latter it is assumed that the hybrids are quark-antiquark states moving on an adiabatic surface generated by an excited flux tube of gluons, with the standard $q \bar{q}$ mesons corresponding to the unexcited flux tube. Decays of hybrids and $q \bar{q}$ mesons then proceed by the same phenomenological pair-creation mechanism, for example the ${ }^{3} P_{0}$ model, coupled with a flux tube overlap [39]. While there are many common features in the decay modes predicted by these approaches there are some substantial differences which become rather crucial in interpreting data, and which are caused partly by the different level of flexibility allowed within the models. In the constituent model the decay strength is proportional to the strong coupling constant $\alpha_{s}\left(q^{2}\right)$ given at some characteristic scale $q^{2}$. As the present level of modeling does not permit definition of this scale, the decay strength was treated as a model parameter in $[24,50]$. The analysis in [24] was based on the assumption of $\hat{\rho}(1400)$ being a hybrid and some upper limits of its $\rho \pi$ mode, which were used as an input to define the decay strength. In the original version [39] of the flux tube model the decay strength was defined from the data on $q \bar{q}$ decays. In principle, in the flux tube model the quark pair creation vertex is uncorrelated with the gluonic modes of the hybrid. This permits the inclusion of different decay vertices within the same overall structure. One has recently been proposed [51], motivated by the heavy-quark limit of the QCD Hamiltonian, and its predictions compared with those of the standard ${ }^{3} P_{0}$ vertex [48]. Once again there are many similarities but some major differences which should be amenable to experimental test although present data cannot distinguish.

The hybrid decays of particular interest to us here are those of the isovector $0^{-+}$and $1^{-+}$, and both the isovector and isoscalar $1^{--}$. There are some substantial differences between the flux tube model $[39,51]$ and the constituent gluon model $[24,50]$ for these.

$0^{-+}$: In the flux tube model, the principal decay modes 
are $\rho \pi$ and, if the hybrid is sufficiently massive, $f_{0}(1370) \pi$. The $\rho \pi$ width in the constituent gluon model is comparable to that of the flux tube model, but it also has a very large $\pi(\pi \pi)_{S}$ width which dominates the decay.

$1^{-+}:$In this case the flux tube model and the constituent gluon model are in reasonable accord. The principal decay modes are $\rho \pi$ and $b_{1} \pi$, with the latter the larger of the two.

$1^{--}$: The flux tube model predicts a rather narrow isovector state, with $a_{1} \pi$ as the dominant mode. In contrast the constituent gluon model predicts a much larger width, still with $a_{1} \pi$ dominant, but with significant $\rho(\pi \pi)_{S}$ and $\omega \pi$ components. For the corresponding isoscalar, the flux tube model predicts a very narrow state decaying almost entirely to $\rho \pi$. Again the constituent gluon model predicts a much larger width, having $\rho \pi$ as the largest decay mode but also with a significant $\omega(\pi \pi)$ fraction.

We would like to comment here on an important point concerning decays which include $(\pi \pi)_{S}$ in the final state. The flux tube calculations do not consider such decays at all, on the grounds that the complicated dynamics of the $(\pi \pi)_{S}$ final state is incompatible with the simple decay chain of $q \bar{q}$ in the ${ }^{3} P_{0}$ state going into $\pi \pi$. It has even been suggested that an effective Lagrangian approach may provide a better simulation of dynamics when the $(\pi \pi)_{S}$ system is involved [52]. Nevertheless, as the ${ }^{3} P_{0} q \bar{q}$ couples strongly to $\pi \pi$, it should participate in the $\pi \pi$ dynamics even if there exists a non- $q \bar{q}$ mechanism which generates this dynamics. So there is no reasons to neglect the decay channels with $q \bar{q}$ in the ${ }^{3} P_{0}$ state, unless the corresponding amplitude is very small per se.

The interaction in the $(\pi \pi)_{S}$ channel is very strong and requires the unitarized coupled channel analysis, but the $\pi \pi$ phase shift can be described with the ${ }^{3} P_{0} q \bar{q}$ as an intermediate state (see the detailed analysis of [53] and a simple model in Appendix A). It is not surprising that a naive quark model, such as the ${ }^{3} P_{0}$ one, fails to describe the low-mass part of the $\pi \pi S$-wave phase shift, where constituent quarks are not the proper degrees of freedom and chiral physics enters the game instead. It still remains an open question of how to incorporate the chiral symmetry constraints into the quark model unless it is done in a purely phenomenological way. In the simple model described in Appendix A the ${ }^{3} P_{0}$ amplitude is modified to interpolate smoothly between the chiral perturbation theory regime with Adler zeros and the confinement regime with string-breaking modeled by the ${ }^{3} P_{0}$ mechanism.

In summary there are two main lines which can be followed.

(i) Hybrids are comparatively light, the $\pi(1300)$ and $\hat{\rho}(1400)$ are hybrid states (or, in the former case, predominantly hybrid) and the mass of the hybrid $\rho_{H}, \omega_{H}$ $\sim 1.6 \mathrm{GeV}$. This would allow strong mixing of the vector hybrids with the radial and orbital excitations of the $\rho$ and $\omega$, but is not compatible with flux tube models.

(ii) Hybrids are comparatively heavy, the $\hat{\rho}(1600)$ is the lightest $1^{-+}$state and the $\pi(1600)$ presumably the corresponding $0^{-+}$hybrid (or at least contains a significant hybrid component). This scenario is compatible with flux tube models, but puts the $1^{--}$vector hybrid mass at $\sim 2.0 \mathrm{GeV}$, making strong mixing with the radial and orbital excitations unlikely.

\section{PSEUDOSCALAR DECAYS TO GROUND STATE PLUS $(\pi \pi)_{S}$}

We have already commented that in the constituent gluon model of hybrids the decays of the $0^{-+}$state is very different from those predicted by the flux tube model. It has been suggested [24] that if the $\hat{\rho}(1405)$ does exist then the $\pi(1300)$ should have a large hybrid admixture and the $\pi(1300)$ decays would then allow a test of the two models. We explore both this hypothesis and the hypothesis that the $\pi(1300)$ is a $q \bar{q}$ state, specifically the first radial $2{ }^{1} S_{0}$ excitation of the $\pi$. For the latter assumption we can use the decays of the $\eta(1295)$ as a control. To begin with we summarize the current experimental situation with respect to the decays of both these mesons.

\section{A. $\eta(1275)$}

The most detailed study of the decay $\eta(1295)$ $\rightarrow \eta(\pi \pi)_{S}$ comes from the E852 charge-exchange reaction $\pi^{-} p \rightarrow \eta \pi^{-} \pi^{+} n$ [54]. The data are sufficiently precise to allow a separation of the $a_{0} \pi$ and $\eta(\pi \pi)_{S}$ decays despite the similarity of these two channels. The $a_{0} \pi / \eta(\pi \pi)_{S}$ branching ratio is estimated to be $0.48 \pm 0.22$, although this may contain a large systematic error due to the difficulty of distinguishing unambiguously between the $a_{0} \pi$ and $\eta(\pi \pi)_{S}$ decays. This ratio disagrees with the GAMS result of 1.86 \pm 0.60 [55], although it also is possibly subject to similar systematic errors. The total width of the $\eta(1295)$ is rather well defined: $66 \pm 13 \mathrm{MeV}$ from $\mathrm{E} 852,53 \pm 6 \mathrm{MeV}$ from Fukui et al. [56]. Assuming that the two results for the $\Gamma\left(a_{0} \pi\right) / \Gamma\left(\eta(\pi \pi)_{S}\right)$ branching ratios give reasonable upper and lower limits, we can conclude that the partial width for $\eta(1295) \rightarrow \eta(\pi \pi)_{S}$ is in the approximate range 20 to 40 $\mathrm{MeV}$.

\section{B. $\pi(1300)$}

Until recently there has been little information on the $\pi(\pi \pi)_{S}$ branching fraction of the $\pi(1300)$, and there is still considerable uncertainty in the total width, which can lie somewhere in the range 200 to $600 \mathrm{MeV}$ [1]. The recent VES data [33,34] show a clear $\pi(1300)$ peak in $3 \pi$, with a width of $\Gamma \sim 400$ to $500 \mathrm{MeV}$ in both $\pi(\pi \pi)_{S}$ and $\pi \rho$. The latter appears particularly strong and it has been suggested [21] that as the size of the Deck background in $\pi(\pi \pi)_{S}$ is uncertain it could provide the totality of the $\pi(\pi \pi)_{S}$ signal. If this is correct then the dominant decay would be $\rho \pi$. In contrast the E852 experiment [57] claims three decay modes of the $\pi(1300): \pi \rho, \pi f_{2}(1270)$ and $\pi(\pi \pi)_{S}$. No comment is made on the mass or width of the $\pi(1300)$ other than it is broad. As for the VES experiment, the genuine resonance signal could be confused by interference with the Deck background.

An uncertain Deck background is not a problem for $p \bar{p}$ annihilation experiments. In their study of $f_{0}(1500)$ decays 
in $4 \pi^{0}$ in $p \bar{p} \rightarrow 5 \pi^{0}$ at rest, the Crystal Barrel experiment [58] found a very substantial improvement in their fit when the $\pi \pi(1300)$ decay of the $f_{0}(1500)$ was included. As the final state in this case is all $\pi^{0}$ the decay of the $\pi(1300)$ cannot be to $\pi \rho$ and must be to $\pi(\pi \pi)_{S}$. However because of the restriction to the purely neutral channel nothing can be said about the $\pi(\pi \pi)_{S}$ branching fraction. The parameters of the $\pi(1300)$, if left free in the fit, are determined to be $M=1.114 \mathrm{GeV}, \Gamma=340 \mathrm{MeV}$. Errors on these are not given. In a preliminary analysis [59] of $\bar{p} p$ and $\bar{p} n$ annihilation to $5 \pi$, the ratio $\left[\pi(1300) \rightarrow \pi(\pi \pi)_{S}\right] /[\pi(1300) \rightarrow \pi \rho]$ is quoted as being not more than $10 \%$. The mass and width of the $\pi(1300)$ are found to be $M=1.400 \pm 0.04 \mathrm{GeV}$ and $\Gamma$ $=275 \pm 50 \mathrm{MeV}$ respectively. The Obelix experiment [60], in their analysis of $p \bar{p} \rightarrow 2 \pi^{+} 2 \pi^{-}$also find a significant improvement in their fit if the $\pi(1300)$ is included in the decay chain. The $\pi(1300)$ parameters are found to be $M$ $=1.275 \pm 0.015 \mathrm{GeV}, \Gamma=218 \pm 100 \mathrm{MeV}$. Both the $\pi(\pi \pi)_{S}$ and $\pi \rho$ modes were required by the fit, and the ratio between them was found to be large: $[\pi(1300)$ $\left.\rightarrow \pi(\pi \pi)_{S}\right] /[\pi(1300) \rightarrow \pi \rho]=5.25 \pm 0.7$. This is appreciably larger than the result $\sim 2.12$ obtained from much earlier data [54]. Thus the situation is very confused with gross discrepancies among the experiments. However it is reasonable to believe that the decay $\pi(1300) \rightarrow \pi(\pi \pi)_{S}$ does exist, but with a completely undetermined branching fraction. It is perhaps significant that the $p \bar{p}$ annihilation experiments find a smaller width than the $\pi p \rightarrow(3 \pi) p$ production experiments, which could be due to the effect of the Deck background on the latter. If this is the case, then a total width of $\sim 300 \mathrm{MeV}$ would seem reasonable, with a partial width for $\pi(\pi \pi)_{S}$ somewhere between 30 and $200 \mathrm{MeV}$.

\section{Pseudoscalar decays in the ${ }^{3} P_{0}$ model}

For this part of the discussion we assume that the $\pi(1300)$ is a $q \bar{q}$ state, specifically the first radial $2{ }^{1} S_{0}$ excitation of the $\pi$. For calculating decays in the ${ }^{3} P_{0}$ model we also assume that the $(\pi \pi)_{S}$ is contained in that model and is the $1{ }^{3} P_{0}$ state. A two-channel model which reproduces the experimental $S$-wave $\pi \pi$ amplitude is described in Appendix A. Both versions of the $(\pi \pi)_{S}$ amplitude described there were used in these calculations i.e. without and with the Adler zero included. The results are very much the same in both cases.

In the ${ }^{3} P_{0}$ model, with standard wave-function parameters, the decay of a radial excitation to the corresponding ground state plus $(\pi \pi)_{S}$ is small, a few $\mathrm{MeV}$ at most. This is true for $\rho_{S}, \omega_{S}, \eta(1295), \pi(1300)$. It is caused by a node in the wave function, with the consequence that the decay is very sensitive to the parameter $\beta$. Of course there are limits within which $\beta$ can vary. A good guide to these limits is provided by [20]. Of the standard decays used to specify $\beta$ and $\gamma, a_{1} \rightarrow \rho \pi$ provides no constraint because of the very large experimental uncertainty on its width; $h_{1} \rightarrow \rho \pi$ provides only a weak constraint $(0.3 \leqslant \beta \leqslant 0.5 \mathrm{GeV})$, again because of experimental uncertainty coupled with a rather weak theoretical dependence on $\beta$; and $b_{1} \rightarrow \omega \pi$ also provides

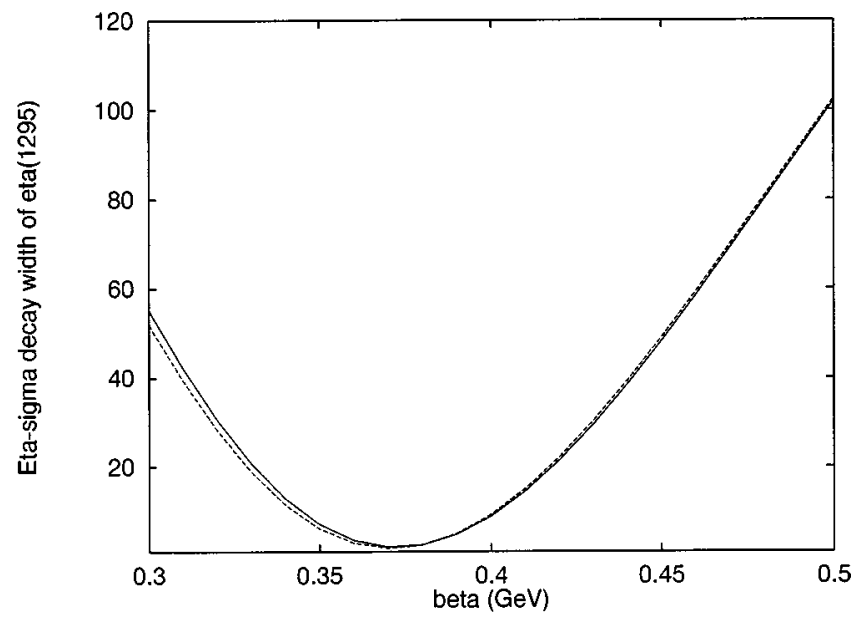

FIG. 1. Width of the decay $\eta^{\prime}(1295) \rightarrow \eta(\pi \pi)_{S}$ as a function of $\beta$. The solid line is calculated using the ${ }^{3} P_{0} \pi \pi$ phase shift and the dashed line using the ${ }^{3} P_{0} \pi \pi$ phase shift modified to include the Adler zero.

only a weak constraint, in this case because the theoretical width is almost independent of $\beta$ over quite a wide range. In contrast the theoretical widths of $f_{2} \rightarrow \pi \pi, a_{2} \rightarrow \rho \pi$ and $\rho$ $\rightarrow \pi \pi$ vary strongly with $\beta$. The two former favor a value of $\beta$ close to the mean of $0.4 \mathrm{GeV}$, but the latter prefers a much smaller value, $\sim 0.3 \mathrm{GeV}$. In contrast the $D / S$ ratios for the decays $b_{1} \rightarrow \omega \pi$ and $a_{1} \rightarrow \rho \pi$, which are sensitive tests of the ${ }^{3} P_{0}$ model, prefer a larger value, particularly the latter, which could go as high as $\beta \sim 0.5 \mathrm{GeV}$. Taking everything into account, $0.3 \mathrm{GeV}$ and $0.5 \mathrm{GeV}$ do seem to provide extreme lower and upper limits on $\beta$, with $0.35 \mathrm{GeV}$ and 0.45 $\mathrm{GeV}$ being more reasonable.

The variation of the $\eta(\pi \pi)_{S}$ width of the $\eta(1295)$ is given in Fig. 1 as a function of the value of $\beta$ for $(\pi \pi)_{S}$. Clearly the experimental limits on the $\eta(1295)$ width provide a strong constraint on the allowed values of $\beta$, independently of other decays. In the ${ }^{3} P_{0}$ model with standard values of the parameters $\beta$ and $\gamma$ the width $\Gamma\left(\pi^{\prime} \rightarrow \pi \rho\right) \sim 200 \mathrm{MeV}$ and the width $\Gamma\left[\pi^{\prime} \rightarrow \pi(\pi \pi)_{S}\right] \sim 0 \mathrm{MeV}$ [21]. The $\pi \rho$ partial width does have quite a strong dependence on $\beta$, decreasing from $\sim 300 \mathrm{MeV}$ at $\beta=0.3 \mathrm{GeV}$ to $\sim 100 \mathrm{MeV}$ at $\beta$ $\sim 0.5 \mathrm{GeV}$ [21]. The variation of the $\pi(\pi \pi)_{S}$ width is much stronger, due to the effect of the node in the wave function. This variation is shown in Fig. 2, where we have taken into account the effect of symmetrization of like pions. The procedure for this is outlined in Appendix B. The constraints imposed by the $\eta(1295)$ decay width clearly restrict the maximum partial width for the $\pi(1300) \rightarrow \pi(\pi \pi)_{S}$ to about $60 \mathrm{MeV}$. This is consistent with the latest result from Crystal Barrel [59], but is appreciably smaller than the result from Obelix [60].

We can conclude the the ${ }^{3} P_{0}$ model cannot be used to explain a very large $\pi(\pi \pi)_{S}$ width of the $\pi(1300)$, should that prove to be required. However a large $\pi(\pi \pi)_{S}$ width of the $\pi(1300)$ does not necessarily provide an argument in favor of a hybrid interpretation and we now consider an alternative non- ${ }^{3} P_{0}$ mechanism. 


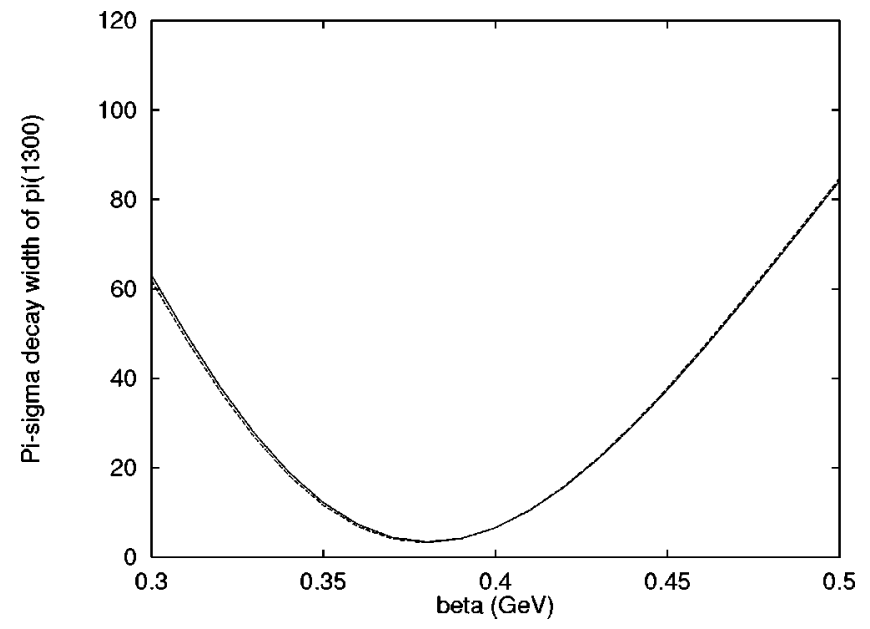

FIG. 2. Width of the decay $\pi(1300) \rightarrow \pi(\pi \pi)_{S}$ as a function of $\beta$. The curves are as in Fig. 1 .

\section{Non- ${ }^{3} P_{0}$ decays of the pseudoscalars}

The decays of heavy-quarkonia radial excitations to the ground state, or to a lower radial, plus $(\pi \pi)_{S}$ raise the possibility of analogous non- ${ }^{3} P_{0}$ decays in the light-quark sector. The detailed mechanism is presumably rather different from that of the models applied to the decays of heavy quarkonia as they rely on a multipole expansion of the gluon field $[61,62]$. The interactions of gluons with a wavelength $\lambda$ much larger than the radius $r_{Q \bar{Q}}$ of quarkonia are suppressed in the multipole expansion by powers of $r_{Q \bar{Q}} / \lambda$. So although it is reasonable to consider only the leading operator for heavy quarkonia it is not so for light quark states.

An alternative mechanism which does not rely on a multipole expansion, "vacuum excitation," has been suggested [63]. This is applicable in principle to both light and heavy quarkonia. The essential idea is that a radial excitation decays to a lower radial excitation or to the ground state by exciting a virtual state from the vacuum into reality. This naturally has the quantum numbers of the vacuum, i.e. $I$ $=0, J=0$ which of course are precisely those of $(\pi \pi)_{S}$. For our present purposes we simplify the calculation by considering relative phase-space as the spatial wave functions of the $\eta(1295)$ and $\pi(1300)$ are identical, and so overlap integrals will be the same. We consider both unweighted and weighted phase space, using for the latter the same $S$-wave $\pi \pi$ amplitude of Appendix A. It is irrelevant whether this is or is not a genuine $q \bar{q}$ state. All that is required is an accurate representation of the amplitude. We find that the ratio of $\left[\pi(1300) \rightarrow \pi(\pi \pi)_{S}\right] /\left[\eta(1295) \rightarrow \eta(\pi \pi)_{S}\right]$ is 3.6 for unweighted phase space and 7.7 for weighted phase space. Thus given a width of 20 to $40 \mathrm{MeV}$ for the $\eta(1295)$ decay it is not difficult to generate the required large width for the $\pi(1300)$ decay.

So we conclude that this particular non- ${ }^{3} P_{0}$ mechanism can correlate the $\eta(1295)$ and $\pi(1300)$ decays and provide a large $\pi(\pi \pi)_{S}$ width for the latter without the need to invoke a hybrid. Of course this remains a hypothesis as we do not have a specific model with which to calculate these decays for light quarks.

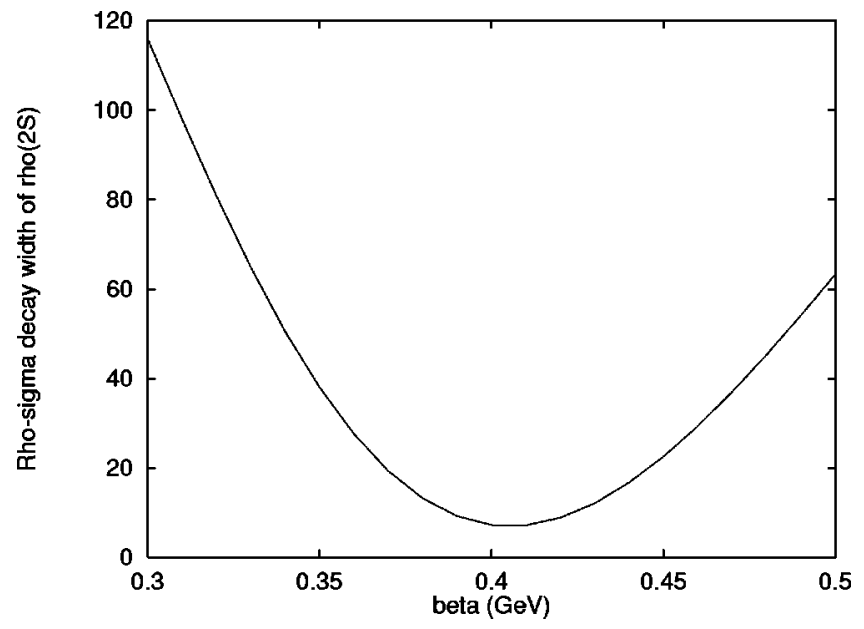

FIG. 3. Width of the decay $\rho(2 S) \rightarrow \rho(\pi \pi)_{S}$ as a function of $\beta$. In contrast to Figs. 1 and 2 only one curve is shown as the results of using the different phase shifts are indistinguishable.

\section{VECTOR STATES}

We now consider the implications of the results of the previous sections for the interpretation of the data from $e^{+} e^{-}$annihilation and $\tau$ decay. We consider two extremes.

(1) The vector hybrids are too heavy to permit significant mixing with the $q \bar{q}$ states in the relevant kinematical region. The $\rho_{S}$ and $\omega_{S}$ decays are some combination of ${ }^{3} P_{0}$ and direct hadronic decay to $\rho(\pi \pi)_{S}$ and $\omega(\pi \pi)_{S}$ respectively. The $\rho_{D}$ and $\omega_{D}$ decays are purely ${ }^{3} P_{0}$. Some mixing between the $2 S$ and $1 D$ states can be allowed.

(2) The vector hybrids are sufficiently light to allow strong mixing with the $q \bar{q}$ states. For completeness we consider the predictions of both the flux tube model and the constituent gluon model for the hybrid decays. However it must be remembered that the flux tube model prefers a higher mass for the vector hybrid.

\section{A. No vector hybrids}

The width of the decay $\rho_{2 S} \rightarrow \rho(\pi \pi)_{S}$ in the ${ }^{3} P_{0}$ model is shown in Fig. 3. Not surprisingly it is too small to account for the observed large $4 \pi$ width of the $\rho(1450)$, even at the maximum acceptable value of $\beta$. To extend the non- ${ }^{3} P_{0}$ decay process suggested in Sec. IV D to the decay of the $\rho_{2 S}$ requires the assumption that only $S$-waves are relevant. This is certainly in accord with the decay of heavy quarkonia. The matrix element for the decay of $\eta(1295)$ or $\pi(1300)$ is simply a constant, $f_{S}$ say. The general form of the matrix element for the decay of $\rho_{2 S}$ is

$$
\begin{aligned}
A_{\mu \nu}= & f\left(-g_{\mu \nu}+\frac{k_{\mu} k_{\nu}}{k^{2}}+\frac{q_{\mu} q_{\nu}}{q^{2}}-\frac{(k q) k_{\mu} q_{\nu}}{k^{2} q^{2}}\right) \\
& +g\left(\frac{(k q) k_{\mu}}{k^{2}}-q_{\mu}\right)\left(k_{\nu}-\frac{(k q) q_{\nu}}{q^{2}}\right)
\end{aligned}
$$

where $k$ and $q$ are respectively the four-momenta of the $\rho_{2 S}$ and the $\rho$. If only $S$-wave is present then 


$$
f=f_{S}, \quad g=-f_{S} / m_{0}\left(m_{1}+E_{1}\right)
$$

where $m_{0}$ and $m_{1}$ are the masses of the $\rho_{2 S}$ and $\rho$, and $E_{1}$ is the energy of the $\rho$ in the $\rho_{2 S}$ rest frame. One has then to calculate $\frac{1}{3} A_{\mu \nu}^{*} A_{\mu \nu}$ which is simply $f_{S}^{2}$. So the only difference between the decays of the vector radial excitations and the pseudoscalar radial excitations is due to phase space. As the available phase space for the decay $\rho_{2 S} \rightarrow \rho(\pi \pi)_{S}$ is not very different from that for the decay $\eta(1295) \rightarrow \eta(\pi \pi)_{S}$, the partial width will be comparable.

Thus neither mechanism by itself can explain the $4 \pi$ decays of the $\rho(1450)$. Nor can one add them to increase the width, as the combined strength is controlled by the $\eta(1295)$ decay. It should be recalled that mixing of the $\rho_{2 S}$ and the $\rho_{1 D}$ is unlikely to resolve the difficulty as, even with an increase in the $\rho(\pi \pi)_{S}$ width of the former within the limits allowed, the $\pi^{+} \pi^{-} \pi^{0} \pi^{0}$ cross section will still exceed the $\pi^{+} \pi^{-} \pi^{+} \pi^{-}$cross section. This conclusion is not affected by adjusting the ${ }^{3} P_{0}$ parameters for the $\rho_{1 D}$ decays, as the $a_{1} \pi$ and $h_{1} \pi$ widths move in unison and the equality remains essentially unchanged. Thus we are forced to conclude that the $e^{+} e^{-} \rightarrow 4 \pi$ data cannot be explained in terms of conventional $q \bar{q}$ dynamics.

The partial width for the decay $\omega_{2 S} \rightarrow \omega(\pi \pi)_{S}$ in either of these models is comparable to that of the corresponding $\rho_{2 S}$ decay. This does not pose any particular problem for the isoscalar sector and does not provide any further insight into the likely mechanisms as it is not nearly sufficient in itself to provide the requisite integrated $\omega \pi \pi$ fraction. Thus we remain with the earlier conclusion that within the isoscalar $q \bar{q}$ structure there must be a significant $\omega_{1 D}$ component.

\section{B. Vector hybrids}

It is now apparent that the inclusion of a isovector vector hybrid is essential to explain the $e^{+} e^{-} \rightarrow 4 \pi$ data, and consequently the corresponding isoscalar vector hybrid must be included in any discussion of the $e^{+} e^{-} \rightarrow \rho \pi, \omega \pi \pi$ data. It is reasonable to assume that the hidden-strange vector hybrid is sufficiently massive not to affect the discussion.

The real question is, what can be inferred about the nature of these hybrids? The flux tube model with its dominant $a_{1} \pi$ decay would appear to resolve the problem in the isovector sector. To achieve strong mixing with the $\rho_{2 S}$ and $\omega_{2 S}$ requires a comparatively low mass. We have seen in Sec. III that the mass scale for the flux tube model is high, making strong mixing unlikely. However spin-dependent forces may lower the mass of the hybrid $\rho$ and $\omega$, which are spin $S=0$ in contrast to the conventional $q \bar{q}$ components which are $S$ $=1$, sufficiently to allow strong mixing between hybrid and conventional quarkonia. There is perhaps less of a problem with the constituent gluon model, but it is still a concern. However accepting the low-mass hybrid scenario, one could then have

$$
|V\rangle=\cos \phi\left\{\cos \theta\left|2{ }^{3} S_{1}\right\rangle+\sin \theta\left|1^{3} D_{1}\right\rangle\right\}+\sin \phi\left|V_{H}\right\rangle .
$$

To explain the predominance of the $\pi^{+} \pi^{-} \pi^{+} \pi^{-}$channel in this model it is necessary to take $\theta \sim 0$, so that $\rho_{1}^{\prime}$ is given by

$$
\left|\rho_{1}^{\prime}\right\rangle \sim \cos \phi\left|\rho_{S}\right\rangle+\sin \phi\left|\rho_{H}\right\rangle
$$

This simple scheme would make the $\pi^{+} \pi^{-} \pi^{+} \pi^{-}$and $\pi^{+} \pi^{-} \pi^{0} \pi^{0}$ widths of the $\rho(1450)$ the same, with only the rather small $\rho(\pi \pi)_{S}$ decay of the $\rho_{H}$ contributing to the observed difference. Further this suppression of the $\rho_{1 D}$ contribution is not compatible with the isoscalar data. Without the $\omega_{1 D}$ there is no source for the strong $\omega \pi \pi$ channel observed experimentally. The $\omega(\pi \pi)_{S}$ decay of $\omega_{H}$ is not sufficient to redress the imbalance. The dynamics would be complex indeed if the ${ }^{3} D_{1}$ state were absent, or nearly so, in the isovector channel but gave a significant contribution in the isoscalar channel. This is perhaps not entirely implausible as the isoscalar channel is complicated by the presence of the $\phi_{2 S}$ right in the middle of the relevant mass range. Hadronic mixing with the nearby $\omega_{2 S}, \omega_{1 D}$ and $\omega_{H}$ will certainly occur at some level, modifying the isoscalar mixing pattern. However this mixing would have to be remarkably strong to produce the differences observed.

The opposite view, that the hybrid mass is high, also does not permit a simple mixing scheme. Mixing essentially between the hybrid and the ${ }^{3} D_{1}$ state is immediately ruled out by the $\rho(1450)$ decays, although it would appear a sustainable option for the isoscalar hadronic decays. Further the $e^{+} e^{-}$widths of the $\rho(1700)$ and $\omega(1650)$ demand mixing with the $\rho_{2 S}$.

The necessity to consider a complicated mixing scheme brings us back to the problem of the hybrid mass scale. The simplest way to achieve strong mixing between $\rho_{2 S}$ and the hybrid is to have them nearly degenerate, which means that the hybrid is very light, about $1.4 \mathrm{GeV}$. Setting the mass scale in such a way opens up exciting possibilities for the spectroscopy of states with nonexotic quantum numbers, along the lines discussed in [24]. It has the additional advantage of being compatible with the sum rules result [41], which we consider as the most reliable of the sum rules analyses. Nevertheless, the exotic $\rho-\pi$ signals at $1.6 \mathrm{GeV}$ from BNL and VES appear sound, and an alternative explanation of the BNL signal at $1.4 \mathrm{GeV}$ has been proposed. Moreover, it is very improbable to have two exotic hybrids so close to each other. Another argument against such a light hybrid comes from the pseudoscalar sector: if there is no need to invoke a hybrid interpretation to explain the decay pattern of $\pi(1300)$, the new pseudoscalars $\pi(1600)$ and $\pi(1800)$ from VES can be economically considered as admixtures of $3 S q \bar{q}$ and hybrid states. It should be remembered that the hybrid interpretation of the $\pi(1800)[21,23]$ emerges from its decay properties.

An alternative scenario is to let the hybrid mix strongly with the $1 D$ state through their near-degeneracy, and then let the lower of the two mixed states mix with the $2 S$. This would mean that the $4 \pi$ decays of the two observed physical states would be quasi-identical. This also suggests that the third state is put rather high and, in addition, does not have much electromagnetic coupling. This scenario assumes the vector hybrid at $\sim 1.7 \mathrm{GeV}$, which makes the mass problem easier to swallow and is compatible with the exotic and pseudoscalar hybrid sector, including the splitting ordering. 
However this scenario is no longer the straightforward two-level mixing scheme initially proposed for the isovector channel. All three states must be included, and in the isoscalar channel one cannot ignore the possible additional complications arising from the $\phi_{2 S}$. The results of such mixing depend on the fine details of the positions of the bare states and the mixing strengths. It is worth mentioning here that one could expect rather strong mixing between quarkonia and hybrids in the constituent model via constituent gluon emission and/or absorption. On the other hand, there are no distinguishable gluons in the flux tube model, and, consequently, no obvious mechanism to provide such mixing.

We have not attempted to construct a detailed mixing scheme here as it requires consideration of all channels and a theoretically-constrained fit to the data [65]. As a first step we present below a simple three-level mixing model which describes qualitatively the isovector data.

\section{A simple mixing scheme}

We consider the mixing of the $2 S$, the $1 D$ and the hybrid $H_{0}$. For the $3 \times 3$ mixing matrix use the standard PDG one without the phase:

$$
\left(\begin{array}{ccc}
c_{12} c_{13} & s_{12} c_{13} & s_{13} \\
-s_{12} c_{23}-c_{12} s_{23} s_{13} & c_{12} c_{23}-s_{12} s_{23} s_{13} & s_{23} c_{13} \\
s_{12} s_{23}-c_{12} c_{23} s_{13} & -c_{12} s_{23}-s_{12} c_{23} s_{13} & c_{23} c_{13}
\end{array}\right) .
$$

Then

$$
\begin{aligned}
\left|\psi_{1}\right\rangle= & c_{12} c_{13}|2 S\rangle+s_{12} c_{13}\left|H_{0}\right\rangle+s_{13}|1 D\rangle \\
|H\rangle= & -\left[s_{12} c_{23}+c_{12} s_{23} s_{13}\right]|2 S\rangle+\left[c_{12} c_{23}\right. \\
& \left.-s_{12} s_{23} s_{13}\right]\left|H_{0}\right\rangle+s_{23} c_{13}|1 D\rangle \\
\left|\psi_{2}\right\rangle= & {\left[s_{12} s_{23}-c_{12} c_{23} s_{13}\right]|2 S\rangle-\left[c_{12} s_{23}\right.} \\
& \left.+s_{12} c_{23} s_{13}\right]\left|H_{0}\right\rangle+c_{23} c_{13}|1 D\rangle .
\end{aligned}
$$

We hypothesize that we want no direct mixing between the $|2 S\rangle$ and the $|1 D\rangle$ so set $s_{13} \sim 0$ and $c_{13} \sim 1$. Thus we have

$$
\begin{aligned}
\left|\psi_{1}\right\rangle= & c_{12}|2 S\rangle+s_{12}\left|H_{0}\right\rangle \\
|H\rangle= & -s_{12} c_{23}|2 S\rangle+c_{12} c_{23}\left|H_{0}\right\rangle \\
& +s_{23}|1 D\rangle \\
\left|\psi_{2}\right\rangle= & s_{12} s_{23}|2 S\rangle-c_{12} s_{23}\left|H_{0}\right\rangle+c_{23}|1 D\rangle .
\end{aligned}
$$

Obviously we identify $\left|\psi_{1}\right\rangle$ with the $\rho(1450)$. In the absence of a proper dynamical model of the mixing we are free to identify $\rho(17000)$ either with $|H\rangle$ or with $\left|\psi_{2}\right\rangle$. For definiteness we choose the former, but the subsequent discussion follows analogously for the latter.

It is reasonable to assume that the bare hybrid $\left|H_{0}\right\rangle$ has no direct electromagnetic coupling. We make no specific assumption on the electromagnetic coupling of the bare $|1 D\rangle$ other than that it should be small compared to the $|2 S\rangle$. The $e^{+} e^{-}$amplitudes for the physical states are

$$
\begin{aligned}
\left\langle e^{+} e^{-} \mid \psi_{1}\right\rangle= & c_{12}\left\langle e^{+} e^{-} \mid 2 S\right\rangle \\
\left\langle e^{+} e^{-} \mid H\right\rangle= & -s_{12} c_{23}\left\langle e^{+} e^{-} \mid 2 S\right\rangle \\
& +s_{23}\left\langle e^{+} e^{-} \mid 1 D\right\rangle \\
\left\langle e^{+} e^{-} \mid \psi_{2}\right\rangle= & s_{12} s_{23}\left\langle e^{+} e^{-} \mid 2 S\right\rangle \\
& +c_{23}\left\langle e^{+} e^{-} \mid 1 D\right\rangle .
\end{aligned}
$$

We see immediately that provided $s_{12} c_{23}>0$ (and $s_{23}>0$ as the electromagnetic coupling of $|1 D\rangle$ has the opposite sign to that of $|2 S\rangle$ ) we will get the correct relative signs of the electromagnetic couplings of the observed states [8]. In the limit of a vanishing electromagnetic width for the $|1 D\rangle$ then we require $s_{12} c_{23}$ to be $\sim \frac{1}{2}$ to agree with the data analyses.

We know that the $\rho(1700)$ has a small $\omega \pi$ width, which puts another constraint on the mixing. The $\omega \pi$ amplitudes are

$$
\begin{aligned}
\left\langle\omega \pi \mid \psi_{1}\right\rangle= & c_{12}\langle\omega \pi \mid 2 S\rangle \\
\langle\omega \pi \mid H\rangle= & -s_{12} c_{23}\langle\omega \pi \mid 2 S\rangle \\
& +s_{23}\langle\omega \pi \mid 1 D\rangle \\
\left\langle\omega \pi \mid \psi_{2}\right\rangle= & s_{12} s_{23}\langle\omega \pi \mid 2 S\rangle \\
& +c_{23}\langle\omega \pi \mid 1 D\rangle .
\end{aligned}
$$

We have already established that $s_{12} c_{23}>0$ and, in principle, that $s_{23}>0$. The latter is important as it allows here some cancellation between the two terms for the $\omega \pi$ decay of the $|H\rangle$. A very small width is thus not ruled out in principle, although in practice it may not be quite so simple as in the ${ }^{3} P_{0}$ model the $|2 S\rangle$ width is very much larger than the $|1 D\rangle$ width.

Finally we look at the $4 \pi$ decays. We can ignore the $a_{1} \pi$ and $h_{1} \pi$ decays of the $|2 S\rangle$ but we can let it have some $\rho(\pi \pi)_{S}$ decay generated by other means. We should also let the bare hybrid have some $\rho(\pi \pi)_{S}$ decay as well. The amplitudes are then

$$
\begin{aligned}
\left\langle a_{1} \pi \mid \psi_{1}\right\rangle= & s_{12}\left\langle a_{1} \pi \mid H_{0}\right\rangle \\
\left\langle a_{1} \pi \mid H\right\rangle= & c_{12} c_{23}\left\langle a_{1} \pi \mid H_{0}\right\rangle \\
& +s_{23}\left\langle a_{1} \pi \mid 1 D\right\rangle \\
\left\langle a_{1} \pi \mid \psi_{2}\right\rangle= & -c_{12} s_{23}\left\langle a_{1} \pi \mid H_{0}\right\rangle \\
& +c_{23}\left\langle a_{1} \pi \mid 1 D\right\rangle
\end{aligned}
$$

and

$$
\begin{aligned}
& \left\langle h_{1} \pi \mid \psi_{1}\right\rangle=0 \\
& \left\langle h_{1} \pi \mid H\right\rangle=s_{23}\left\langle h_{1} \pi \mid 1 D\right\rangle \\
& \left\langle h_{1} \pi \mid \psi_{2}\right\rangle=c_{23}\left\langle h_{1} \pi \mid 1 D\right\rangle
\end{aligned}
$$


and

$$
\begin{aligned}
\left\langle\rho(\pi \pi)_{S} \mid \psi_{1}\right\rangle= & \left\langle\rho(\pi \pi)_{S} c_{12} \mid 2 S\right\rangle+s_{12}\left\langle\rho(\pi \pi)_{S} \mid H_{0}\right\rangle \\
\left\langle\rho(\pi \pi)_{S} \mid H\right\rangle= & -s_{12} c_{23}\left\langle\rho(\pi \pi)_{S} \mid 2 S\right\rangle \\
& +c_{12} c_{23}\left\langle\rho(\pi \pi)_{S} \mid H_{0}\right\rangle \\
\left\langle\rho(\pi \pi)_{S} \mid \psi_{2}\right\rangle= & s_{12} S_{23}\left\langle\rho(\pi \pi)_{S} \mid 2 S\right\rangle \\
& -c_{12} S_{23}\left\langle\rho(\pi \pi)_{S} \mid H_{0}\right\rangle .
\end{aligned}
$$

None of these are qualitatively inconsistent with observation. They all seem reasonable, particularly the lack of any $h_{1} \pi$ decay of $\left|\psi_{1}\right\rangle$. Note that $\left|\psi_{2}\right\rangle$ has a non-zero $e^{+} e^{-}$width, and is presumably somewhere "off-stage." There is some evidence for isovector states in the vicinity of $2.0 \mathrm{GeV}$ which decay strongly into $6 \pi$ [64]. One of these could be the missing member of the trio.

\section{CONCLUSIONS}

Our general conclusion is that the $e^{+} e^{-}$annihilation and $\tau$ decay data require the existence of a "hidden" vector hybrid in both the isovector and isoscalar channels. The argument is based strongly on the pattern of the observed decays to $\rho(\pi \pi)_{S}$ for the isovectors and to $\omega(\pi \pi)_{S}$ for the isoscalars. The strong mixing evident from the electromagnetic widths is also a key feature. The mixing required is nontrivial, involving the first radial and the first orbital $q \bar{q}$ excitations and the ground-state vector hybrid. Before coming to our general conclusion we explored the limits of the ${ }^{3} P_{0}$ model and proposed a specific non- ${ }^{3} P_{0}$ model in an unsuccessful attempt to explain these data without going beyond the $q \bar{q}$ sector.

More specifically we are inclined towards the constituent gluon model rather than the flux tube model to describe the characteristics of the hybrids. This is based on two aspects: the constituent gluon model can more readily encompass a hybrid mass in the relevant range; and the hybrid decays in the constituent gluon model are the more compatible with the data. It is interesting to note that there are indications in lattice gauge theories in the heavy-quark sector [67] that at $Q \bar{Q}$ separations of less than $2 \mathrm{fm}$ treating glue as string fluctuations is doubtful i.e. the flux tube is not an appropriate picture. Whether these results are straightforwardly applicable to the light quark sector is of course an open question.

The suggestion that the ground state vector hybrid is comparatively light, i.e. about $1.6 \mathrm{GeV}$, has major consequences. Given the hybrid spin-parity sequence discussed in Sec. III, namely $0^{-+}<1^{-+}<1^{--}$, it reopens the question of a hybrid component in the $\pi(1300)$ [24] and emphasizes the urgency of clarifying the status of the $\hat{\rho}(1405)$. For the former it is essential to resolve the current major experimental discrepancies on the $\pi(\pi \pi)_{S}$ width, and for the latter to disprove the possibility that it may be an artifact of a strongly coupled threshold $[35,36]$.

For the vector mesons there are three key requirements. The first is to identify the missing partner of the $\rho(1450)$ and $\rho(1700)$, and equally that of the $\omega(1420)$ and $\omega(1600)$, which are predicated to exist on the basis of hybrid mixing. The second is to establish the decay modes of these vector states, particularly the specific content of the $4 \pi$ channel for the $\rho(1450)$ and $\rho(1700)$ and of the $5 \pi$ channel for the $\omega(1420)$ and $\omega(1600)$. The third is to obtain a better understanding of the corresponding $\phi$ states, of which rather little is known at present.

The obvious place to study the vector mesons is in $e^{+} e^{-}$ annihilation, and DA $\Phi$ NE would be an ideal facility in its higher energy mode. Photoproduction and/or electroproduction provide another possibility, and the higher energies proposed for TJNAF would make this an excellent source. Diffractive production is unlikely to be the dominant mechanism at TJNAF energies, so the vector mesons will not be produced in a clean environment. However this is not necessarily negative, as interference with known states can provide important phase information and other states of interest in the hybrid story e.g. the $\pi(1300)$ and the $\hat{\rho}(1405)$, if it is genuine, will also be produced at these intermediate energies $[68,69]$. The expected continuation of the VES program will provide information on a wide range of mesonic states and could resolve the problem of the $\pi(1300)$ decays and of the existence of the $\hat{\rho}(1405)$.

\section{ACKNOWLEDGMENTS}

This research is supported in part by PPARC, by grant RFBR 96-15-96740 and by the EEC-TMR Program (contract NCT98-0169). We are grateful to Frank Close for helpful discussions.

\section{APPENDIX A: THE $(\pi \pi)_{S}$ AMPLITUDE}

The simple ${ }^{3} P_{0}$ model for the $\pi \pi S$-wave amplitude assumes that the scattering takes place via $0^{++}$intermediate states. There are two such states in this case, one is $(1 / \sqrt{2})(u \bar{u}+d \bar{d})$ and the other is $s \bar{s}$.

The $\pi \pi$ phase shift $\delta$ and the inelasticity $\eta$ are given by

$$
\begin{gathered}
\tan (2 \delta)=\frac{a b_{1}-a_{1} b}{a a_{1}+b b_{1}}, \\
\eta^{2}=\frac{\left(a a_{1}+b b_{1}\right)^{2}+\left(a b_{1}-a_{1} b\right)^{2}}{a^{2}+b^{2}},
\end{gathered}
$$

where

$$
\begin{gathered}
\left.a=a(s)=1+\frac{1}{\left(s-s_{1}\right)\left(s-s_{2}\right)}\left[\left(\operatorname{Im} \Delta_{12}\right)^{2}-\operatorname{Im} \Delta_{11} \operatorname{Im} \Delta_{22}\right)\right], \\
b=b(s)=\frac{1}{s-s_{1}} \operatorname{Im} \Delta_{11}+\frac{1}{s-s_{2}} \operatorname{Im} \Delta_{22}, \\
a_{1}=a_{1}(s)=1+\frac{1}{\left(s-s_{1}\right)\left(s-s_{2}\right)}\left[\left(\operatorname{Im} \widetilde{\Delta}_{12}\right)^{2}\right. \\
\left.\quad-\operatorname{Im} \tilde{\Delta}_{11} \operatorname{Im} \tilde{\Delta}_{22}\right],
\end{gathered}
$$




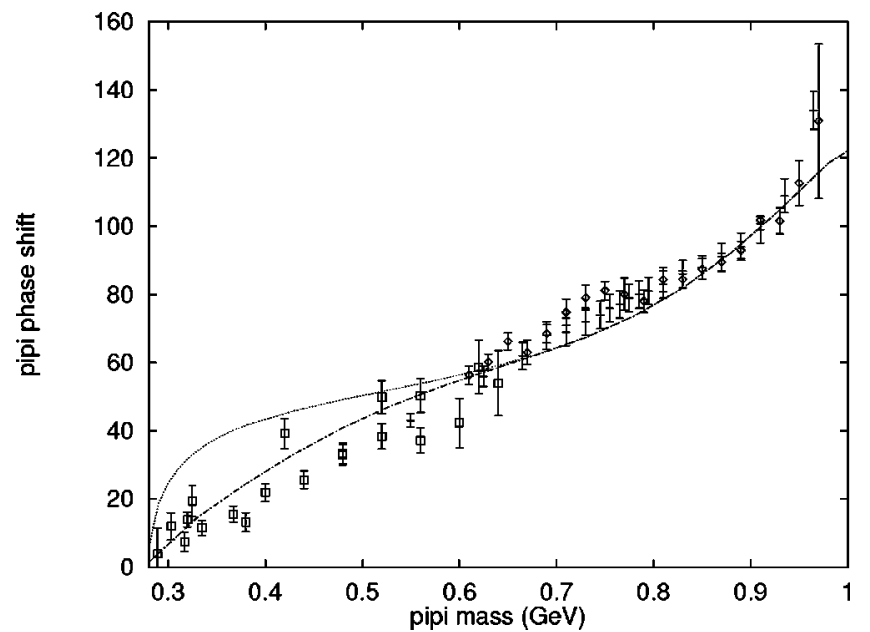

FIG. 4. The $S$-wave $\pi \pi$ phase shift. The solid curve is the result of the ${ }^{3} P_{0}$ calculation and the dashed curve is the result of the low-energy modification to include the Adler zero. The data are from [66].

$$
b_{1}=b_{1}(s)=\frac{1}{s-s_{1}} \operatorname{Im} \tilde{\Delta}_{11}+\frac{1}{s-s_{2}} \operatorname{Im} \tilde{\Delta}_{22} .
$$

In these formulas $\sqrt{s_{1}}$ and $\sqrt{s_{2}}$ are the masses of intermediate states, and

$$
\begin{gathered}
\operatorname{Im} \Delta_{a b}=\sum_{j} \operatorname{Im} \Delta_{a b}(j), \\
\operatorname{Im} \widetilde{\Delta}_{a b}=\operatorname{Im} \Delta_{a b}-2 \operatorname{Im} \Delta_{a b}(\pi \pi),
\end{gathered}
$$

where $a, b=1,2$ label the intermediate states, and $j$ $=\pi \pi, K \bar{K}$ are the coupled channels taken into account.

The quantities $\operatorname{Im} \Delta_{a b}(j)$ are calculated in the framework of ${ }^{3} P_{0}$ model as

$$
\operatorname{Im} \Delta_{a b}(j)=\frac{p_{j}}{8 \pi \sqrt{s}} f_{a}(j) f_{b}(j)
$$

with the ${ }^{3} P_{0}$ form factors $f_{a}(j)$ defined as in [18].

This model is an oversimplified version of a coupled channel method and preserves unitarity but not analyticity; in contrast to the much more sophisticated model [53] the physical resonance positions $\sqrt{s_{a}}$ are taken to be constants and are not defined via dispersion relations.

The $\pi \pi S$-wave phase shift given by the ${ }^{3} P_{0}$ model is shown as the solid line in Fig. 4. To improve the agreement with the data close to the $\pi \pi$ threshold the Adler zero is now introduced. We make it pragmatically by substituting

$$
f_{a}(\pi \pi) \rightarrow \widetilde{f}_{a}(\pi \pi)=f_{a}(\pi \pi) F(s)
$$

with $F(s)$ being a smooth function of $s . \quad F\left(m_{\pi}^{2} / 2\right)=0$, $F(s) \rightarrow 1$ when $s$ increases. In such a way we have the Adler zero at $s_{0}=m_{\pi}^{2} / 2$ in the $\pi \pi$ amplitude as required by chiral symmetry. The $\pi \pi S$-wave phase shift with this modification is shown as the dashed line in Fig. 4 for the simple choice

$$
F(s)=\theta\left(s_{1}-s\right)\left[-\left(\frac{\sqrt{s}-\sqrt{s_{0}}}{\sqrt{s_{1}}-\sqrt{s_{0}}}\right)^{2}+2\left(\frac{\sqrt{s}-\sqrt{s_{0}}}{\sqrt{s_{1}}-\sqrt{s_{0}}}\right)\right]
$$

with $\sqrt{s_{1}}=0.7 \mathrm{GeV}$ so that chiral dynamics and quark dynamics are matched at $0.7 \mathrm{GeV}$.

The idea to insert Adler zeroes into the quark model form factors was suggested in [53]. Our procedure differs from that one. In contrast to [53] we believe that the soft pion physics governs only the lower end of the $\pi \pi$ mass spectrum and does not affect the whole mass range available, leaving room for the strong interaction of quarks at higher energies. Moreover it is our belief that with the more QCD-motivated model for hadronic decays [48], which takes into account the Goldstone nature of pions, the quark model description is valid at lower $\pi \pi$ masses as well. This makes the Adler zero constraints responsible for only a rather small energy range just above the $\pi \pi$ threshold.

\section{APPENDIX B: THE PIPRIME DECAY WIDTH}

The width of the decay of $\pi^{\prime 0}$ into $\pi^{+} \pi^{-} \pi^{0}$ is given by

$$
\begin{gathered}
d \Gamma=\frac{2}{3} \frac{1}{(2 \pi)^{3}} \frac{1}{32 m_{\pi^{\prime}}^{3}}\left|M_{c}\right|^{2} d s_{12} d s_{13} \\
\left|M_{c}\right|^{2}=D
\end{gathered}
$$

and the width into three neutral pions is

$$
\begin{gathered}
d \Gamma=\frac{1}{3} \frac{1}{(2 \pi)^{3}} \frac{1}{32 m_{\pi^{\prime}}^{3}}\left|M_{0}\right|^{2} d s_{12} d s_{13} \\
\left|M_{0}\right|^{2}=D+2 I \\
D=f_{\pi^{\prime}}^{2}\left(s_{12}\right) f_{\sigma}^{2}\left(s_{12}\right) P\left(s_{12}\right) \\
f_{\pi^{\prime}}\left(s_{12}\right) f_{\pi^{\prime}}\left(s_{13}\right) f_{\sigma}\left(s_{12}\right) f_{\sigma}\left(s_{13}\right) P\left(s_{12}\right) P\left(s_{13}\right) \\
\times\left[\left(s_{12}-m_{\sigma}^{2}\right)\left(s_{13}-m_{\sigma}^{2}\right)+\operatorname{Im}\left(s_{12}\right) \operatorname{Im}\left(s_{13}\right)\right] \\
f_{\pi^{\prime}}(s)=f_{\pi^{\prime} \sigma \pi}\left(m_{\pi^{\prime}}^{2}, s, m_{\pi}^{2}\right) \\
f_{\sigma}(s)=f_{\sigma \pi \pi}\left(s, m_{\pi}^{2}, m_{\pi}^{2}\right) \\
P(s)=\left[\left(s-m_{\sigma}^{2}\right)^{2}+\operatorname{Im}^{2}(s)\right]^{-1}
\end{gathered}
$$




$$
\operatorname{Im}(s)=\frac{1}{8 \pi \sqrt{s}} p\left(s, m_{\pi}^{2}, m_{\pi}^{2}\right) f_{\sigma}^{2}(s) .
$$

The total widths of these decays are given by

$$
\begin{gathered}
\Gamma=\int_{s_{12 \min }}^{s_{12 \max }} \int_{s_{13 \min }}^{s_{13 \max }} d \Gamma \\
s_{13 \max }=\frac{m_{\pi^{\prime}}^{2}+3 m_{\pi}^{2}-s_{12}}{2} \\
+2 \sqrt{\frac{\left(s_{12}-4 m_{\pi}^{2}\right)}{4} \frac{\left(m_{\pi^{\prime}}^{2}-m_{\pi}^{2}-s_{12}\right)^{2}-4 m_{\pi^{2}}^{2} s_{12}}{4 s_{12}}}
\end{gathered}
$$

$$
\begin{aligned}
s_{13 \min }= & \frac{m_{\pi^{\prime}}^{2}+3 m_{\pi}^{2}-s_{12}}{2} \\
& -2 \sqrt{\frac{\left(s_{12}-4 m_{\pi}^{2}\right)}{4} \frac{\left(m_{\pi^{\prime}}^{2}-m_{\pi}^{2}-s_{12}\right)^{2}-4 m_{\pi}^{2} s_{12}}{4 s_{12}}}
\end{aligned}
$$$$
s_{12 \max }=\left(m_{\pi^{\prime}}-m_{\pi}\right)^{2}
$$$$
s_{12 \min }=4 m_{\pi}^{2}
$$

[1] Particle Data Group, C. Caso et al., Eur. Phys. J. C 3, 415 (1998).

[2] L. M. Barkov et al., Nucl. Phys. B256, 365 (1985); D. Bisello et al., Phys. Lett. B 220, 321 (1989).

[3] S. I. Dolinsky et al., Phys. Lett. B 174, 453 (1986); D. Bisello et al., in Proceedings of the XXV ICHEP, Singapore, 1990, edited by K. K. Phua and Y. Yamaguchi (World Scientific, Singapore, 1992).

[4] ALEPH Collaboration, R. Barate et al., Z. Phys. C 76, 15 (1997); CLEO Collaboration, L. P. Perera, in Proceedings of HADRON'97, BNL, 1997, edited by S-U Chung and H. J. Willutski (American Institute of Physics, New York, 1998), p. 595.

[5] ARGUS Collaboration, H. Albrecht et al., Phys. Lett. B 185, 223 (1987).

[6] S. I. Dolinsky et al., Phys. Rep. 202, 99 (1991); DM2 Collaboration, L. Stanco, in Proceedings of HADRON'91, Maryland, 1991, edited by Y. Oneda and D. Peaslee (World Scientific, Singapore, 1992).

[7] C. Bacci et al., Nucl. Phys. B184, 31 (1981); G. Cosme et al., ibid. B152, 215 (1979).

[8] A. B. Clegg and A. Donnachie, Z. Phys. C 62, 455 (1994); A. Donnachie and A. B. Clegg, Phys. Rev. D 51, 4979 (1995).

[9] N. N. Achasov and A. A. Kozhevnikov, Phys. Rev. D 55, 2663 (1997).

[10] A. Antonelli et al., Phys. Lett. B 212, 133 (1988).

[11] S. Fukui et al., Phys. Lett. B 202, 133 (1988).

[12] Crystal Barrel Collaboration, A. Abele et al., Phys. Lett. B 391, 191 (1997); Crystal Barrel Collaboration, U. Thoma, in Proceedings of HADRON'97, BNL, 1997, edited by S-U Chung and H. J. Willutski (American Institute of Physics, New York, 1998), p. 322; Crystal Barrel Collaboration, A. Abele et al., Phys. Lett. B 450, 275 (1999).

[13] A. Antonelli et al., Z. Phys. C 56, 15 (1992).

[14] Crystal Barrel Collaboration, R. McCrady, in Proceedings of HADRON'97, BNL, 1997, edited by S-U Chung and H. J. Willutski (American Institute of Physics, New York, 1998), p. 331.

[15] LASS Collaboration, D. Aston et al., Nucl. Phys. B (Proc. Suppl.) 21, 105 (1991).

[16] S. Godfrey and N. Isgur, Phys. Rev. D 32, 189 (1985).
[17] G. Busetto and L. Oliver, Z. Phys. C 20, 247 (1983); P. Geiger and E. S. Swanson, Phys. Rev. D 50, 6855 (1994); H. G. Blundell and S. Godfrey, ibid. 53, 3700 (1996).

[18] R. Kokoski and N. Isgur, Phys. Rev. D 35, 907 (1987).

[19] A. LeYaouanc, L. Oliver, O. Pene, and J. Raynal, Phys. Rev. D 8, 2223 (1973).

[20] E. S. Ackleh, T. Barnes, and E. S. Swanson, Phys. Rev. D 54, 6811 (1996).

[21] T. Barnes, F. E. Close, P. R. Page, and E. S. Swanson, Phys. Rev. D 55, 4157 (1997).

[22] A. Donnachie and Yu. S. Kalashnikova, Z. Phys. C 59, 621 (1993).

[23] F. E. Close and P. R. Page, Phys. Rev. D 56, 1584 (1997).

[24] Yu. S. Kalashnikova, Z. Phys. C 62, 323 (1994).

[25] L. S. Kisslinger, J. Gardner, and C. Vanderstraeten, Phys. Lett. B 410, 1 (1997).

[26] L. S. Kisslinger and W-h. Ma, hep-ph/9905479.

[27] E852 Collaboration, D. P. Weygand, in Proceedings of HADRON'97, BNL, edited by S-U Chung and H. J. Willutski (American Institute of Physics, New York, 1998), p. 313.

[28] VES Collaboration, Yu. P. Gouz, in Proceedings of the XXVI ICHEP, Dallas, 1992, edited by J. R. Sanford, p. 572.

[29] E852 Collaboration, D. Ryabchikov, in Proceedings of HADRON'97, BNL, edited by S-U Chung and H. J. Willutski (American Institute of Physics, New York, 1998), p. 527.

[30] P. R. Page, Phys. Lett. B 415, 205 (1997).

[31] E852 Collaboration, D. R. Thompson et al., Phys. Rev. Lett. 79, 1630 (1997).

[32] Crystal Barrel Collaboration, A. Abele et al., Phys. Lett. B 423, 175 (1998).

[33] VES Collaboration, A. Zaitsev, in Proceedings of HADRON'97, BNL, 1997, edited by S-U Chung and H. J. Willutski (American Institute of Physics, New York, 1998), p. 461; VES Collaboration, D. V. Amelin, ibid., p. 770.

[34] VES Collaboration, D. V. Amelin et al., Phys. Lett. B 356, 595 (1995).

[35] A. Donnachie and P. R. Page, Phys. Rev. D 58, 114012 (1998).

[36] A. Donnachie and Yu. S. Kalashnikova (in preparation).

[37] T. Barnes and F. E. Close, Phys. Lett. 116B, 365 (1982); 
123B, 89 (1983); T. Barnes, F. E. Close, and F. de Viron, Nucl. Phys. B224, 241 (1983).

[38] M. Chanowitz and S. Sharpe, Nucl. Phys. B222, 211 (1983).

[39] N. Isgur and J. E. Paton, Phys. Lett. 124B, 247 (1983); N. Isgur, R. Kokoski, and J. E. Paton, Phys. Rev. Lett. 54, 869 (1985); N. Isgur and J. E. Paton, Phys. Rev. D 31, 2910 (1985); T. Barnes, F. E. Close, and E. S. Swanson, Phys. Rev. D 52, 5242 (1995).

[40] Yu. S. Kalashnikova and Yu. B. Yufryakov, Phys. Lett. B 359, 175 (1995); Yu. S. Kalashnikova and Yu. B. Yufryakov, Yad. Fiz. 60, 374 (1997) [Phys. At. Nucl. 60, 307 (1997)].

[41] I. I. Balitsky, D. I. Dyakonov, and A. V. Yung, Z. Phys. C 33, 265 (1986).

[42] J. I. Latorre, P. Pascual, and S. Narison, Z. Phys. C 34, 347 (1987).

[43] C. McNeile, hep-lat/9904013.

[44] P. Lacock and K. Schilling, Nucl. Phys. B (Proc. Suppl.) 73, 261 (1999).

[45] C. Bernard et al., Phys. Rev. D 56, 7039 (1997); Nucl. Phys. B (Proc. Suppl.) 73, 264 (1999).

[46] P. Lacock, C. Michael, P. Boyle, and P. Rowland, Phys. Lett. B 401, 308 (1997).

[47] P. R. Page, hep-ph/9806233, Proceeding of MESON'98, Cracow, Poland.

[48] A. P. Szczepaniak and E. S. Swanson, Phys. Rev. D 55, 3987 (1997).

[49] UKQCD Collaboration, P. Lacock et al., Phys. Rev. D 54, 6997 (1996); Phys. Lett. B 401, 308 (1997).

[50] F. Iddir, A. Le Yaouanc, L. Oliver, O. Pène, and J. C. Raynal, Phys. Lett. B 205, 564 (1988); A. Le Yaounac, L. Oliver, O. Pène, J. C. Raynal, and S. Ono, Z. Phys. C 28, 309 (1985).

[51] P. R. Page, E. S. Swanson, and A. P. Szczepaniak, Phys. Rev. D 59, 034016 (1999).

[52] F. E. Close, in Proceedings of HADRON'97, BNL, edited by
S-U Chung and H. J. Willutski (American Institute of Physics, New York, 1998), p. 892.

[53] N. A. Tornqvist, Z. Phys. C 68, 647 (1995).

[54] J. J. Manak, Ph.D. thesis, Notre Dame University.

[55] GAMS Collaboration, D. Alde et al., Yad. Fiz. 60, 458 (1997) [Phys. At. Nucl. 60, 386 (1997)].

[56] A. Fukui et al., Phys. Lett. B 267, 293 (1991).

[57] E852 Collaboration, D. Weygand, in Proceedings of HADRON'95, Manchester, 1995, edited by M. Birse, G. Lafferty, and J. McGovern (World Scientific, Singapore, 1996), p. 241.

[58] Crystal Barrel Collaboration, A. Abele et al., Phys. Lett. B 380, 453 (1996).

[59] Crystal Barrel Collaboration, U. Thoma, Proceedings of the Workshop on Hadron Spectroscopy, Frascati, 1999.

[60] Obelix Collaboration, A. Bertin et al., Phys. Lett. B 414, 220 (1997).

[61] R. Aaron and R. S. Longacre, Phys. Rev. D 24, 1207 (1981).

[62] K. Gottfried, Phys. Rev. Lett. 40, 538 (1978); T.-M. Yan, Phys. Rev. D 22, 1652 (1980); Y.-P. Kuang and T.-M. Yan, ibid. 24, 2874 (1981); J.-W. Chen and M. J. Savage, ibid. 57, 2837 (1998).

[63] A. Donnachie and N. Isgur (private communication).

[64] A. B. Clegg and A. Donnachie, Z. Phys. C 45, 677 (1990).

[65] A. B. Clegg, A. Donnachie, and Yu. S. Kalashnikova (in preparation).

[66] S. D. Protopopescu et al., Phys. Rev. D 7, 1279 (1973); B. Hyams et al., Nucl. Phys. B64, 134 (1973); L. Rosselet et al., Phys. Rev. D 15, 574 (1977).

[67] K. J. Juge, J. Kuti, and C. J. Morningstar, Nucl. Phys. B (Proc. Suppl.) 73, 590 (1999); hep-lat/9809211.

[68] A. Afanasev and P. R. Page, Phys. Rev. D 57, 6771 (1998).

[69] A. Donnachie and Yu. S. Kalashnikova (in preparation). 